\title{
Design of Spectrum Database Assisted Cognitive Radio Vehicular Networks
}

\author{
Rahman Doost-Mohammady and Kaushik Roy Chowdhury \\ Department of Electrical and Computer Engineering, Northeastern University, Boston, USA \\ Email: $\{$ doost,krc $\} @$ ece.neu.edu
}

\begin{abstract}
Recent FCC rulings in the US, mandating spectrum databases for white spaces unlicensed access, raise interesting challenges on the placement of road-side base stations (BS) connected to spectrum databases distributing spectrum knowledge to cognitive radios embedded in passing by vehicles. This paper takes the first step towards developing an analytical framework for determining the topology of such a vehicular network, where the network components are the spectrum database, multiple BSs and moving vehicles in different traffic conditions. Our framework not only adheres to the FCC specifications, but it also provides a cost-revenue analysis given the environmental parameters such as vehicular density, BS road coverage and the tradeoffs between local vs. database assisted sensing, thereby making this a potential tool for future network design. A comprehensive simulation study is undertaken using real traffic traces to show the application and benefit of our method in a practical setting.
\end{abstract}

\section{INTRODUCTION}

The recent rulings by the FCC mandating the creation of spectrum databases [1] have reduced the erstwhile complete dependence on spectrum sensing alone to infer the primary user (PU) activity in the licensed bands. Now, cognitive radio (CR) devices can query dedicated databases for correct and updated knowledge of the actively used channels in their neighborhood. While this is a significant step towards realizing practical CR implementations, the new resulting network architecture and associated connectivity rules raise several challenges in the seamless operation of the network. This paper takes the first steps towards an analytical formulation for the placement of spectrum database query points, and is aimed to serve as a design tool for creating such database assisted networks for mobile or vehicular scenarios. We analyze in depth the cost-benefit tradeoff of the resulting network, while factoring in diverse considerations of vehicular density, road dimensions, querying costs, permissible error bounds, among others.

Location awareness, mobility, and spectrum database access capability are the three defining parameters that give rise to the various modes of operation defined by the FCC in the US in 2010 [1]. These network components are shown in Figure 1 for a road length $L$, and explained in further detail below:

- Fixed TV Band devices (TVBDs) are installed at a fixed location and their coordinates are known to themselves and to the public, e.g., BSs $\mathrm{X}$ and $\mathrm{Y}$.

- Mode II device is portable and has internal geolocation capabilities, along with the access capability to the spectrum database.

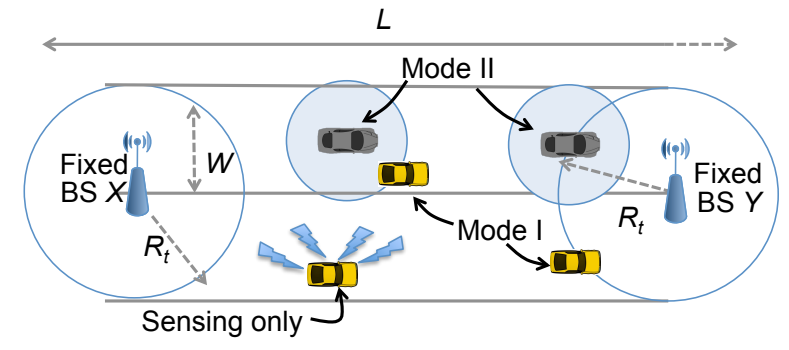

Fig. 1. Different modes of operation in a vehicular CR network

- Mode I is a portable device with no geolocation capability and no direct access to the database. This type of device must contact its neighboring Mode II or Fixed device to obtain the list of available TV channels.

- sensing-only is a FCC certified portable device unable to access the spectrum database through either a Fixed or Mode II device, and must rely on local spectrum measurements.

We assume in this paper that vehicles are equipped with (i) geolocation ability through GPS (or any other form of accurate localization tool), (ii) 3G connectivity that allows sending queries requesting spectrum information, and (iii) local spectrum sensing hardware, with varying probabilities. A combination of these features directly result in the mode of operation described above. As an example, a device with geolocation and 3G connectivity assumes the role of a Mode II device. The main contribution in this paper is the formulation of an analytical optimization framework that gives the covered percentage of the road by fixed BSs spread uniformly for a given length of a roadway, while taking into consideration a probabilistic estimate of the CR devices that facilitate database access, and those who must rely on such services. This framework finds the optimal solution subject to the constraints of permissible error in estimating the available spectrum and more than zero revenue received at each BS, while minimizing the cost of operation of the network.

The rest of this paper is organized as follows. We give the related work in this area and establish the background for the modeling in Section II. In Section III, we describe our optimization framework in detail, and provide supporting analysis. We undertake a thorough performance evaluation in Section IV, and finally, Section V concludes our work. 
TABLE I

EMPIRICALLY MEASURED TRAFFIC PARAMETERS FROM [4]

\begin{tabular}{|l|l|l|l|l|}
\hline Time of Day & Traffic type & $\begin{array}{l}\lambda_{s} \\
{[\text { veh/m }]}\end{array}$ & $\begin{array}{l}\lambda_{t} \\
{[\text { veh/s] }]}\end{array}$ & $\begin{array}{l}\text { fit goodness } \\
{[\%]}\end{array}$ \\
\hline 01:00 am $-03: 00 \mathrm{am}$ & low & 0.0039 & 0.1192 & $(2.65,2.42)$ \\
10:00 am $-12: 00 \mathrm{pm}$ & normal & 0.0250 & 0.7276 & $(3.13,9.27)$ \\
15:00 pm - 17:00 pm & high & 0.0728 & 0.7813 & $(8.09,9.67)$ \\
\hline
\end{tabular}

\section{Related Work And Model Preliminaries}

The opening of White spaces for unlicensed use is expected to create a boost across all sectors of the economy in the coming years [2]. This is due to the fact that a lot of applications could benefit from the availability of white spaces. CR vehicular networks constitute an emerging application area for opportunistic access of licensed spectrum for vehicle to vehicle communication, streaming entertainment, and various driver-assist services [3]. Recent measurement data collected in [4] on a single day on the 5-lane I-80 highway between Emeryville, CA and Berkeley, CA, provided information on various aspects of vehicular traffic, including inter-vehicle spacing, inter-arrival time and vehicle speed. In our analysis, we rely on the availability of such information for a given length of the road, which can be gathered from off-line studies. Here, inter-vehicle spacing is the distance of any arbitrary car in the road to its closest car in any lane of the road, irrespective of their respective directions. Inter-arrival time is the arrival time of any car at the observation point relative to the arrival of the previous car. The result of the data analysis in [4] suggests that inter-vehicle spacings and inter-arrival times are exponentially distributed. However during low traffic time when the traffic is less than $1000 \mathrm{veh} / \mathrm{hr}$, the accuracy of the fit is the highest. Table II shows the exponential mean $\lambda_{s}$ and $\lambda_{s}$ for inter-vehicle spacing and inter-arrival time at 3 different times of the day outlined in [4], which we use in our analysis.

\section{Proposed Optimization Framework FOR BS PLACEMENT}

The overall aim of this analysis is to provide guidelines for the uniform placement of the BSs that will provide information about the availability of DTV channels, and thereby support the mobile CR enabled vehicles. The BSs are fixed, directly connected to spectrum databases, and have transmission radius of $R_{t}$. The actual number of deployed BSs along the road directly influences the density of deployment $\alpha$. Our optimization framework determines this $\alpha$ so as to minimize the cost of spectrum information access and also maximize the accuracy of spectrum information, irrespective of the method it is obtained either from the database or via local spectrum sensing (in the event where getting the database information is impossible).

We assume that each CR user operates in a saturated state, constantly looking for free white space to transmit data to peers. To help access the spectrum information, each device can be potentially equipped with three different options, i.e. (i) Spectrum Sensing Module, (ii) Internet connection through
3G, 4G or other related technologies, and (iii) GPS for accurate location determination at each point in time, as shown in Table III with independent probabilities $x_{1}$, and $x_{2}$, and $x_{3}$, respectively. Depending on the available options on each CR device, and also its position on the road relative to other network components, we identify the operation of the device under various classes as follows:

Case I. In case I where none of the three options are available for the CR, the device can only be in Mode I. So it has to get spectrum information from nearby CR devices in Mode II or, when in range, from a Fixed BS. Probability of having at least one Mode II CR device in range around an arbitrary node is given by Lemma 1:

Lemma 1. If the inter-vehicle spacing has an exponential distribution of mean $\lambda_{s}$, and probability of a vehicle being in Mode $\Delta$ is $P_{\Delta}$, then the probability of having at least one Mode II device in range $R$ is $1-e^{P_{\Delta} R \lambda_{s}}$.

Proof: With an exponential distribution of the intervehicle spacing with mean $\lambda_{s}$, the number of vehicles in range $\mathrm{R}$ of an arbitrary vehicle has a poisson distribution with mean $R \lambda_{s}$ [6]. Thus, the probability of having at least one vehicle in Mode II, is calculated as follows:

$$
A=\sum_{i=1}^{\infty} \frac{\left(R \lambda_{s}\right)^{i} e^{-\left(R \lambda_{s}\right)}}{i !}\left[1-\left(1-P_{\Delta}\right)^{i}\right]
$$

In the above equation, the probability of having at least one Mode $\Delta$ device is $\left(1-\left(1-P_{\Delta}\right)^{i}\right)$, given $i$ nodes in the range. Using taylor series expansion, $A$ can be reduced to:

$$
\begin{aligned}
A & =e^{-\left(R \lambda_{s}\right)}\left[\sum_{i=1}^{\infty} \frac{\left(R \lambda_{s}\right)^{i}}{i !}-\sum_{i=1}^{\infty} \frac{\left(\left(1-P_{\Delta}\right) R \lambda_{s}\right)^{i}}{i !}\right] \\
& =e^{-\left(R \lambda_{s}\right)}\left[e^{R \lambda_{s}}-1-e^{\left(1-P_{\Delta}\right) R \lambda_{s}}+1\right] \\
& =1-e^{-P_{\Delta} R \lambda_{s}}
\end{aligned}
$$

Given Case I, probability of being Mode I, is:

$$
P_{M I \mid \text { CaseI }}=\alpha+(1-\alpha) A
$$

Additionally, we choose to call the condition when the $\mathrm{CR}$ user is not any of the FCC specified modes (i.e., I, II or sensing-only), as the silent mode. With respect to case Case I, such a probability is derived as:

$$
P_{\text {silent } \mid \text { CaseI }}=1-\alpha-(1-\alpha) A
$$

Case II. In this case only the GPS is installed and no Internet connection exists. Consequently, the CR device is in Mode II only when it is in the range of a BS or yet another Mode II device, from which it can obtain spectrum database access. When no database access by any means is possible, the CR device is technically in Mode I. However, since the FCC rulings do not permit the sharing of channel information among other Mode I devices, the CR user must go into silent 


\begin{tabular}{|c|c|c|c|c|c|}
\hline $\begin{array}{l}\text { Vehicle Equipment } \rightarrow \\
\downarrow \text { Probability of Occurrence }\end{array}$ & $\begin{array}{l}\text { Spectrum } \\
\text { Sensing } \\
x_{1}\end{array}$ & $\begin{array}{l}\text { Internet } \\
\text { Connection } \\
x_{2}\end{array}$ & $\begin{array}{l}\text { Geolocation } \\
x_{3}\end{array}$ & Device Mode & $\begin{array}{c}\text { Case } \\
\text { Number }\end{array}$ \\
\hline$\left(1-x_{1}\right)\left(1-x_{2}\right)\left(1-x_{3}\right)$ & - & - & - & Mode I when connected to BS or a Mode II, otherwise silent & I \\
\hline$\left(1-x_{1}\right)\left(1-x_{2}\right) x_{3}$ & - & - & $\sqrt{ }$ & Mode II when connected to BS or a Mode II, otherwise silent & II \\
\hline$\left(1-x_{1}\right) x_{2}\left(1-x_{3}\right)$ & - & $\sqrt{ }$ & - & Mode I when connected to BS or a Mode II, otherwise silent & III \\
\hline$\left(1-x_{1}\right) x_{2} x_{3}$ & - & $\sqrt{ }$ & $\sqrt{ }$ & always in Mode II & IV \\
\hline$x_{1}\left(1-x_{2}\right)\left(1-x_{3}\right)$ & $\sqrt{ }$ & - & - & Mode I when connected to BS or a Mode II, otherwise Sensing-Only & $\mathrm{V}$ \\
\hline$x_{1}\left(1-x_{2}\right) x_{3}$ & $\sqrt{ }$ & - & $\sqrt{ }$ & Mode I when connected to BS or a Mode II, otherwise Sensing-Only & VI \\
\hline$x_{1} x_{2}\left(1-x_{3}\right)$ & $\sqrt{ }$ & $\sqrt{ }$ & - & Mode I when connected to BS or a Mode II, otherwise Sensing-Only & VII \\
\hline$x_{1} x_{2} x_{3}$ & $\sqrt{ }$ & $\sqrt{ }$ & $\sqrt{ }$ & always in Mode II & VIII \\
\hline
\end{tabular}

mode until it moves to a position where database access is possible. Probability of being in Mode II, in this case is:

$$
P_{M I I \mid \text { CaseII }}=\alpha+(1-\alpha) A
$$

Note that the probability of silent mode is similar to that in Case I.

Case III. The CR user is in Mode I and the probability of silent mode similar to Case I.

Case IV. In this case, where both internet connection and GPS is available, the CR user is always in Mode II.

$$
P_{M I I \mid \text { CaseIV }}=1
$$

Case V. In this case, only spectrum sensing module is installed, the CR user switches between Mode I and sensing-only modes. Mode I occurs when there's a nearby BS or Mode II device to transfer the information, and otherwise it becomes sensing-only. The probabilities of Mode I and sensing-only correspond to probabilities of Mode I and silent mode in Case I respectively.

Case VI. Here, both GPS and the sensing module are installed, and the device switches between Mode II and sensing-only mode. The probabilities of Mode II and sensing-only corresponds to probabilities of being in Mode II and silent mode in Case II, respectively.

Case VII. In this case, the CR user switches between Mode $\mathrm{I}$ and sensing-only mode similar to case $\mathrm{V}$. The probability of being in each mode is exactly the same as Case V.

Case VIII. Finally, in case VIII, the CR user is always in Mode II, the same as Case IV.

Summing up on the Mode I probabilities on different cases, the cumulative probability of a CR user being in Mode I is:

$$
\begin{aligned}
P_{M I} & =P_{M I \mid C a s e I} P_{\text {CaseI }}+P_{M I \mid C a s e I I I} P_{\text {CaseIII }} \\
& +P_{M I \mid C a s e V} P_{\text {CaseV }}+P_{M I \mid C a s e V I I} P_{\text {CaseVII }} \\
& =[\alpha+(1-\alpha) A]\left(1-x_{3}\right)
\end{aligned}
$$

Similarly, the probabilities of being in Mode II, sensing-only mode, and silent mode, respectively, are:

$$
\begin{aligned}
P_{M I I}^{1} & =\left(1-x_{2}\right) x_{3}[\alpha+(1-\alpha) A] \\
P_{M I I}^{2} & =x_{2} x_{3} \\
P_{M I I} & =\left(1-x_{2}\right) x_{3}[\alpha+(1-\alpha) A]+x_{2} x_{3} \\
P_{\text {sense }} & =x_{1}\left(1-x_{2} x_{3}\right)[1-\alpha-(1-\alpha) A] \\
P_{\text {silent }} & =\left(1-x_{1}\right)\left(1-x_{2} x_{3}\right)[1-\alpha-(1-\alpha) A]
\end{aligned}
$$

Here, $P_{M I I}^{1}$ is the probability of being a Mode II with no direct Internet access, but through another Mode II or fixed BS. $P_{M I I}^{2}$ is the probability of Mode II with direct access.

A Mode II CR user with no direct database access potentially acts as relay node to forward queries from another Mode II CR user to a nearby fixed BS, or a nearby Mode I device. Since the chain of Mode II relay nodes can induce delay to the spectrum information queries originating from a Mode II or Mode I CR user, we put a constraint on the number of relay nodes for both limiting the delay and also for the sake of analysis simplicities. The constraint is that a Mode II device with no direct database access can only relay data to a mode I CR user and only when it's connected to a BS. Given this constraint, $P_{\Delta}$ in equation (2) will be $x_{2} x_{3}+\left(1-x_{2}\right) x_{3} \alpha$. Also looking at direct-access and BS-access mode cases separately, $P_{\Delta}$ must be replaced with $x_{2} x_{3}$ and $\left(1-x_{2}\right) x_{3} \alpha$ :

$$
\begin{aligned}
& A^{1}=1-e^{-\left(1-x_{2}\right) x_{3} \alpha R \lambda_{s}} \\
& A^{2}=1-e^{-x_{2} x_{3} R \lambda_{s}}
\end{aligned}
$$

where $A^{1}$ and $A^{2}$ are the probabilities of having at least one Mode II device in the range of a BS-assisted database access and an arbitrary node with direct database access respectively.

The cost of channel information access depends on the source of the channel information. We assume for each query the requesting CR user will be charged $s_{1}$ by the Fixed BSs and $s_{2}$ by the directly connected Mode II CR users.

Hence, the cost of channel access for users in Mode I is:

$$
C_{M I}=\alpha s_{1}+(1-\alpha)\left[A^{1} s_{1}+\left(1-A^{1}\right) A^{2} s_{2}\right]
$$

The first term in the equation above is for the time when Mode I device is in the range of a BS. The second term, however, considers the probability that there is a Mode II device, with preferably indirect (because of lower cost) or direct access, in the range of the requesting Mode I device. The cost of channel access for Mode II users with BS access is the same as Mode I users' access cost, and the cost of channel access for users in Mode II with direct access is $s_{2}$.

The average cost of channel information access for users can be expressed in general terms as follows:

$$
C_{\text {user }}=P_{M I} C_{M I}+P_{M I I}^{1} C_{M I I}^{1}+P_{M I I}^{2} C_{M I I}^{2}
$$

The average revenue received for a channel information query made by an arbitrary user is:

$$
C_{R e v}=\left[P_{M I}+P_{M I I}^{1}\right] \alpha s_{1}
$$


The density of fixed base stations will directly determine the average cost of channel access for a road of given traffic type. The optimized value for minimized cost can be determined by the following optimization problem:

$$
\begin{aligned}
& \text { Given : } R, \lambda_{s}, x_{1}, x_{2}, x_{3}, s_{1}, s_{2} \\
& \text { To find }: \alpha \\
& \text { To Minimize }: C_{\text {user }} \\
& \text { Subject to : } \\
& P_{e} \leq P_{e}^{\text {lim }} \\
& C_{\text {Rev }} N_{\text {av }}-C_{\text {annual }} \geq 0
\end{aligned}
$$

- The probability of sensing error $P_{e}$, due to spectrum sensing can be calculated as follows:

$$
\begin{aligned}
& P_{e}=\quad P_{\text {sense }}\left[P_{m d}+P_{f a}\right] \\
& =x_{1}\left(1-x_{2} x_{3}\right)[1-\alpha-(1-\alpha) A]\left[P_{m d}+P_{f d}(20)\right.
\end{aligned}
$$

The constraint (18) limits this error within a threshold $P_{e}^{l i m}$, where $P_{m d}$ and $P_{f a}$ are the probabilities of missed detection and false alarm, respectively.

- In constraint (19), $N_{a v}$ is the average number of vehicles querying a specific fixed BS on the road and is calculated as follows:

$$
N_{a v}=\lambda_{t}^{a v} \times 86400 \times 365 \times\left[P_{M I}+P_{M I I}^{1}\right]
$$

where $\lambda_{t}^{a v}$ is the average inter-arrival time at any BS along the road. This is with the assumption that every Mode I and Indirect Mode II vehicle will ping the BS once it passes by it. Also $C_{\text {annual }}$ in (19) is the annual cost of maintaining a fixed BS on the road. In here we only consider the maintenance cost of each BS, since it accounts for the major part of the BS cost (around 75\% [5]).

The derivative of $C_{u s e r}$ can help solve the above optimization problem. Simple derivation will lead to:

$$
\begin{aligned}
C_{\text {user }}^{\prime} & =\left(1+(1-\alpha)\left(1-x_{2}\right) x_{3} R \lambda_{s}\right)\left[\left(s_{1}-s_{2}\right) \alpha\right. \\
& \left.+(1-\alpha)\left(2 A^{1} s_{1}+A^{2} s_{2}\right)\right] A \\
& +\alpha s_{2} A^{2}+(1-\alpha) s_{2} A A^{2}
\end{aligned}
$$

In the next section, we numerically show that the derivation of $C_{\text {user }}$ is always positive, so the function has its minimum point at one of the two ends of $\alpha$ range which is confirmed by the simulation result of the cost function.

\section{Performance Evaluation}

In this section, we perform a numerical analysis of the optimization framework given in the previous section and show how different metrics vary with the increasing BS density. We simulate the given mathematical formulation within MATLAB while fixing inputs of the optimization problem as shown in table IV. Parameter $s_{2}$ is the cost of internet access through $3 \mathrm{G}$ or similar technologies and its value is calculated based on the current price of $\$ 25$ for $2 G B$ data traffic and a exchange of $20 \mathrm{~KB}$ data at each incident of database access. Also $s_{1}$
TABLE II

SIMULATION PARAMETERS

\begin{tabular}{|l|l|}
\hline Parameter & Value \\
\hline$x_{1}$ & $5 \%$ \\
$x_{2}$ & $20 \%$ \\
$x_{3}$ & $40 \%$ \\
$P_{m d}$ & 0.05 \\
$P_{f a}$ & 0.05 \\
$R$ & $500 m$ \\
$s_{1}$ & 0.0125 cent \\
$s_{2}$ & 0.025 cent \\
\hline
\end{tabular}

is assumed to be half of $s_{2}$. Probability of mis-detection and false alarms for TV channel sensing are taken to be both 0.05 .

Figure 2 shows the average cost of access to spectrum information per request for different traffic situations. Interestingly, in low and normal traffic scenarios, the cost of access goes up with increasing BS density. This can be observed from Figure 3 , where for these two traffic types, the silent mode probability in low BS density is relatively high. Moreover, the increase of BS density only helps increasing the number of requests to BS, while the likelihood of finding a direct-access Mode II device is low, although it costs more than a BS to retrieve spectrum information from these devices. In high traffic scenarios, the probability for finding a Mode II device is high, and this helps keeping the silent time low, even if the BS density is low as well. Figure 2 also confirms the argument made in the previous section about the local minimum at either end of the cost function domain. Figure 4 shows the derivation of $C_{u s e r}(\alpha)$ function in different traffic types which is positive all over the range as argued in the previous section.

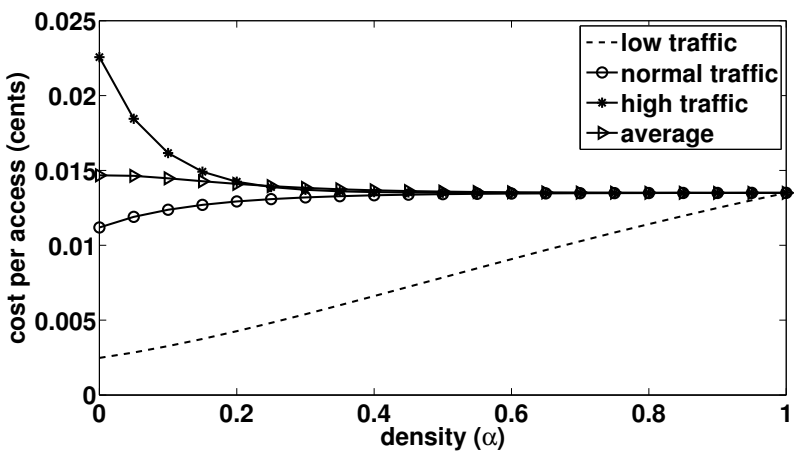

Fig. 2. cost of each channel information access vs. BS density

Figure 5 shows the variation of average sensing error probability occurred with different BS density. It is observed that this probability in low traffic types is significantly higher than other traffic situations, since more of Sensing-only mode will be experienced.

Figures 6 shows the average number of queries made to each BS given the density of BS in the road. Also Figure 7 shows the annual revenue received from the operation of each $\mathrm{BS}$, given the BS density on the road.

The minimum feasible value for $\alpha$ can be determined by setting the right thresholds for the constraints in (18) and (19) and even additional ones may be incorporated that are imperative from a network designer's point of view. 


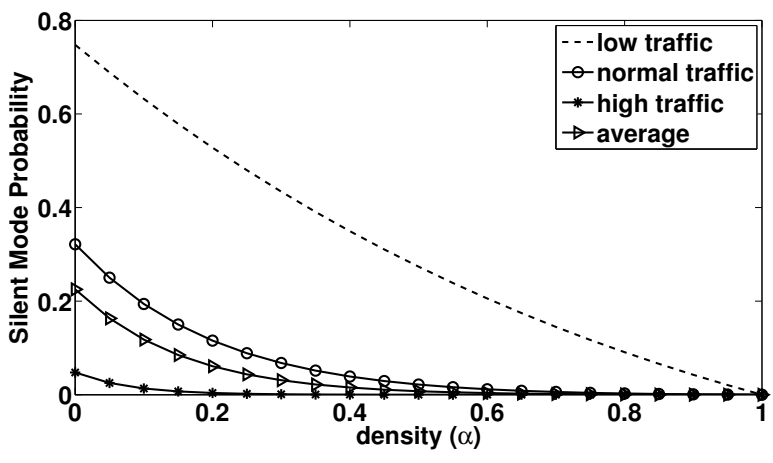

Fig. 3. silent mode probability change vs. BS density

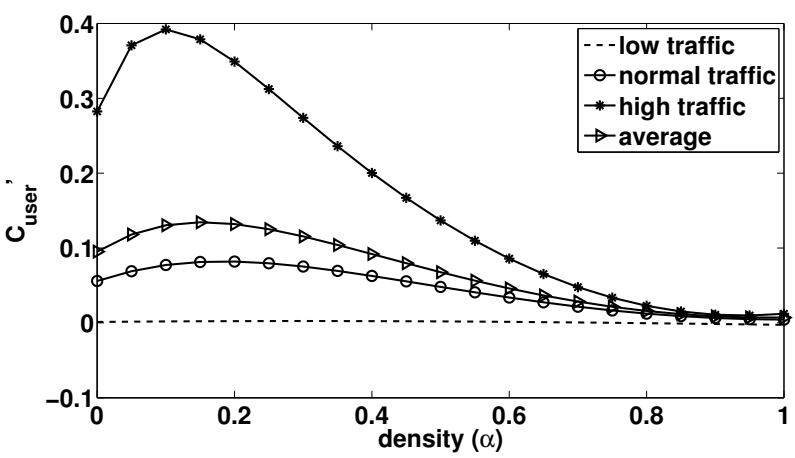

Fig. 4. Access cost function derivation vs. BS density

\section{CONCLUSiON}

We presented a framework for the placement of BSs, given the diverse capabilities of geolocation, querying, and local sensing capabilities that vehicles may be equipped with. The framework effectively minimizes the cost of operation, and ensures the resulting error in spectrum detection stays within bounds. To the best of our knowledge, this is the first work that directly combines the FCC regulations with the concerns of vehicular CR networks. We propose to pursue experimental evaluation of this framework by collecting traces of traffic data and spectrum availability to demonstrate the practical implementation of such a database assisted CR operation.

\section{REFERENCES}

[1] FCC Press Release, 2011. [Online]. Available: http://www.fcc.gov/Daily Releases/Daily Business/ 2011/db0126/DA-11-131A1.pdf.

[2] Microsoft Report, 2009. The economic value generated by current and future allocations of unlicensed spectrum. [Online]. Available: http://www.spectrumbridge.com/Libraries/White Space Primer/ whitespaces-microsoft-study.sflb.ashx.

[3] M. D. Felice, R. Doost-Mohammady, K. R. Chowdhury, and L. Bononi. Smart Radios for Smart Vehicles: Cognitive Vehicular Networks. IEEE Vehicular Technology Magazine - special issue on Applications of Cognitive Radio Networks), to appear, 2012.

[4] N. Wisitpongphan, F. Bai, P. Mudalige, V. Sadekar, and O. Tonguz. Routing in Sparse Vehicular Ad Hoc Wireless Networks. IEEE J. on Selected Areas in Communications, Vol. 25, No. 8, October 2007.

[5] K. Johansson, A. Furuskar, P. Karlsson, J. Zander. Relation between Base Station Characteristics and Cost Structure in Cellular Systems. Personal, Indoor and Mobile Radio Communications, 15th IEEE International Symposium on, September 2004.

[6] A. Popoulis. Probability, Random Variables and Stochastic Processes. Mcgraw-Hill College; 3rd edition.

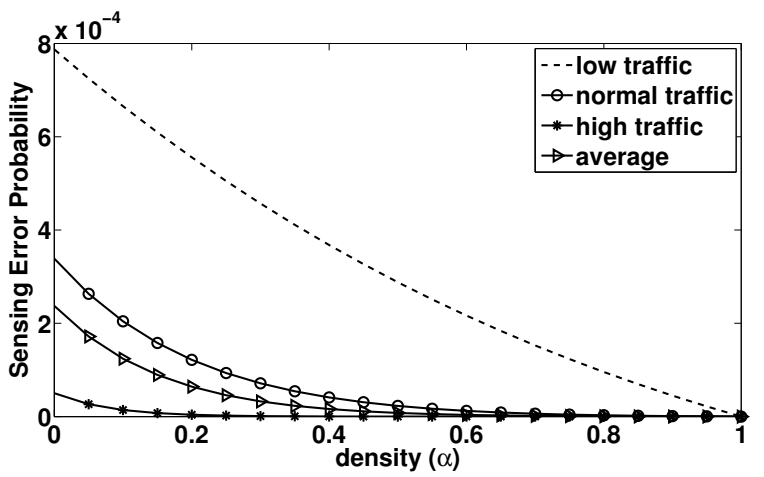

Fig. 5. average sensing error probability vs. BS density

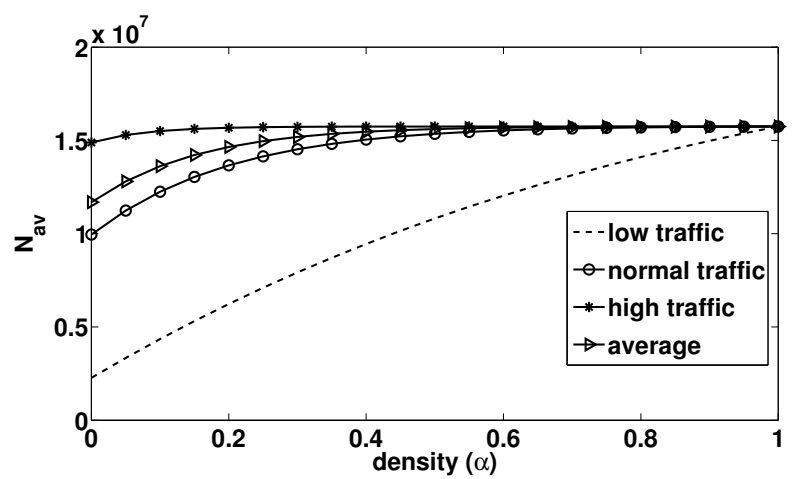

Fig. 6. Average number of channel information accesses per year vs. BS density

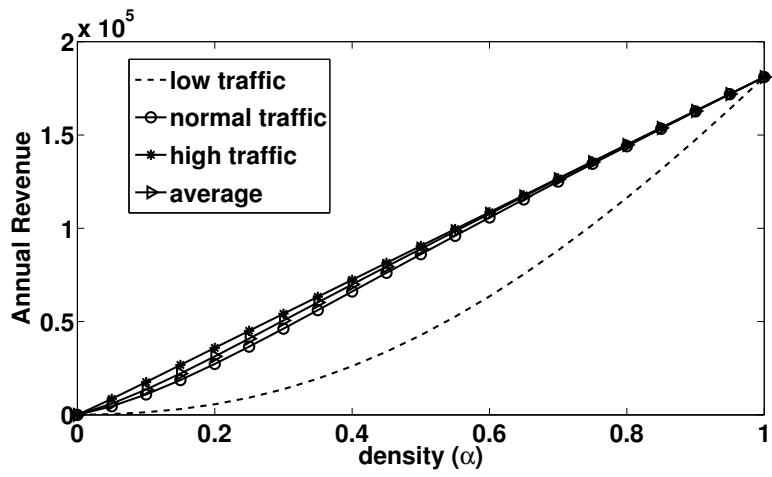

Fig. 7. Average annual revenue received at each BS vs. BS density 